\title{
DYNAMICS AND CLUSTERS OF GALAXIES
}

\author{
D. G. SAAR I \\ Dept. of Mathematics, Northwestern University, Evanston, Ill. 60201, U.S.A.
}

\begin{abstract}
Under the assumption that the inverse square central force law is a good approximation to the gravitational force, at least for large distances, the different possibilities for the evolution of the Universe are sketched. Several of the possibilities lead naturally to a dynamical classification of clusters of galaxies in an expanding universe. In one of the classifications the galaxies must define configurations which are functions of the masses. The virial theorem approach of determining masses of galaxies in a cluster is briefly examined. Some tentative statements concerning a dynamical explanation of the local hypothesis for quasars are advanced. Finally, the role of mathematical probability in predicting the behavior of the Universe is discussed.
\end{abstract}

\section{Introduction}

In earlier papers (Saari, 1971a, b to be referred to as Papers 1 and 2) the general qualitative and asymptotic behavior as time approaches infinity of all solutions of the $n$-body problem was derived and outlined. Here $n$ is an arbitrary but fixed positive integer. That is, in these papers we gave a mathematical description of Newton's universe for large values of time. In this talk I would like to review some of these results, but attempt to do so in a fashion where the mathematical solutions are related to possible astronomical interpretations. That is, we assume that Newton's law is a good approximation for the gravitational force, and then we describe the evolution of the Universe.

In the first sections we shall concentrate on giving different possible dynamical interpretations for clusters of galaxies. This discussion includes a disintegrating system as one type of 'cluster of galaxies'. As a byproduct of this discussion, we shall make some brief comments about the related problem of determining masses of the clusters via the virial theorem.

It will turn out that in one classification of clusters of galaxies the galaxies must tend toward the vertices of well-defined configurations. These configurations are determined by the masses of the galaxies. In some settings the fact that these configurations are functions of the masses may be exploited to yield a method which would either determine whether a cluster is complete (and if not, it would give a prediction scheme which would indicate possible locations where one would expect to find a member galaxy), or determine the masses of the member galaxies up to certain proportionality parameters.

With a partial solution of the equations of motion at hand, a natural question would be to ask if there is anything in the dynamics of the $n$-body problem comparable to the behavior of quasars, namely, can quasars be explained in terms of Newtonian mechanics? In Section 4, we shall offer some extremely tentative statements.

This classification of motion is the result of a mathematical study of the equations of motion of the inverse square central force law for $\boldsymbol{n}$ point masses. However, any realistic model for the Universe would include forces other than the gravitational attraction between masses. In fact, some of the forces probably would dominate the 
gravitational force for 'local' distances. Fortunately, the results given here are stable respect to perturbations of this type. The conclusions stated here hold equally well for any force law which is dominated by the inverse square term for large distances. That is, it holds for force laws of the form

$$
\mathbf{f}(\mathbf{r}, \mathbf{v}, t)=\mu r^{-3} \mathbf{r}+\boldsymbol{\varepsilon}(\mathbf{r}, \mathbf{v}, t),
$$

where $r^{2}|\varepsilon(\mathbf{r}, \mathbf{v}, t)| \rightarrow 0$ as $r \rightarrow \infty$, and $\varepsilon(-\mathbf{r}, \mathbf{v}, t)=-\varepsilon(\mathbf{r}, \mathbf{v}, t)$.

Notice that for local distances the dominating force could be most anything, even a repulsive force law. Indeed, it need not define a conservative system. Also, note that we require the forces to start acting like a central force law only for 'large distances'. In fact, the results hold with only minor modifications even when the inverse square term is changed to an inverse $q$ force law where $1<q<3$. Consequently, the conclusions of this study apply to models which allow for oblateness effects, approximations to relativity, and some nongravitational forces. The major requirement is that the resulting differential equations have unique solutions which exist for $t \geqslant 0$.

\section{Clusters of Galaxies}

It follows from Kepler's equations that one of three things can occur in the two-body problem as time approaches infinity: (a) the motion is bounded, (b) the motion is parabolic, where the distance between particles separates like $t^{2 / 3}$, and (c) the motion is hyperbolic, where the distance between particles separates like $t$.

To see this, recall that the conservation of energy integral for the two-body problem is $\mathbf{v}^{2}=2\left(\mu r^{-1}+h\right)$, where $\mathbf{r}$ and $\mathbf{v}$ are respectively the position vector and velocity vector of the second particle relative to the first. If constant $h$ is negative (elliptic motion), then $\mu r^{-1}+h \geqslant 0$, or $r \leqslant \mu h^{-1}$. This is conclusion (a).

Define $I=r^{2}$. Then $\ddot{I}=2\left(\mathbf{v}^{2}+\mathbf{r} \cdot \ddot{\mathbf{r}}\right)$. From the conservation of energy integral and the equations of motion, this equation can be expressed as

$$
\ddot{I}=2\left(\mu r^{-1}+2 h\right)=2 \mu I^{-1 / 2}+4 h .
$$

If constant $h$ is positive (hyperbolic motion), then $\ddot{I} \geqslant 4 h$. By integrating both sides of this inequality twice we have that $I \geqslant 2 h t^{2}+0\left(t^{2}\right)$. This means that $\ddot{I}=4 h+0\left(t^{-1}\right)$. By integrating this last expression twice we obtain $r^{2}=I=2 h t^{2}+0(t \ln t)$. This is conclusion (c).

Finally, if $h=0$ (parabolic motion), then $\ddot{I}=2 \mu I^{1 / 2}>0$. If $I$ were bounded above for all positive time, then we see from this last inequality that $\ddot{I}$ would be bounded below by a positive constant. By integrating this new inequality twice, we obtain the contradiction that if $I$ is bounded, then it goes to infinity faster than some constant multiple of $t^{2}$. Therefore, we conclude that $I$ is unbounded. However, from the facts that $\ddot{I}$ is positive and $I$ is unbounded we can show that $I \rightarrow \infty$ and that after some time $\dot{I}$ is positive. Therefore, by integrating $\dddot{I} I=2 \mu I^{-1 / 2} \dot{I}$, we see that $\dot{I}^{2}=8 \mu I^{1 / 2}+c$, where $c$ is a constant of integration. Since $I \rightarrow \infty$ and $\dot{I}$ is positive, this can be expressed as $\dot{I} I^{-1 / 4}=(8 \mu)^{1 / 2}+0(1)$ as $t \rightarrow \infty$. Integrating this last expression leads to conclusion (b). 
In the $n$-body problem the motion is, as one would expect, more complicated. If any two particles are chosen from the $n$ particles, then their mutual distance either behaves as described in (a), (b), or (c), or it belongs to two other possible types of motion as given in Paper 1. These other types of motion will be discussed in Section 4. However, as shown in Papers 1 and 2, the important fact is that all solutions of the n-body problem which exist for all positive time (Saari, 1971c) consist of various combinations of these five types of motion. This is independent of the value of the total energy of the system!

For the remainder of this section we will concentrate on those important solutions where the motion is a combination of cases (a), (b), and (c). That is, the distance between any two particles is bounded, expanding like $t^{2 / 3}$, or expanding like $t$. (In the general $n$-body problem the terms 'parabolic' and 'hyperbolic' seem to be out of place, so we drop them and identify the motion via its major characteristic - the distances separate respectively like $t^{2 / 3}$ or like $t$.)

While this description is in terms of the behavior of any two particles, it can be translated immediately into a discussion of the total system. What happens is that several masses may have their mutual distances bounded after some time. These masses define, in a natural fashion, a group. Any two particles chosen from different groups must separate either like $t^{2 / 3}$, or like $t$. Since the groups remain (by construction) relatively bounded entities, it follows that the separation between the two groups in question is respectively like $t^{2 / 3}$ or like $t$. The separation can be measured from the centers of mass of the groups. Also, since $t^{2 / 3} / t \rightarrow 0$ as $t \rightarrow \infty$, after some time a clear distinction between the two rates of expansion would appear. A restricted case of this general picture can be found in Figure 1.

From these three types of motion there are seven qualitatively different possible classes of solutions. They are found from the various possible combinations, and they range from the case where the distances between all particles remain bounded for all time to the case where all three types of motion occur. The discussion of these cases is somewhat similar, and to eliminate repetition only the inclusive solution, which exhibits all three types of motion, will be discussed in detail. A similar discussion would follow for the remaining six types of solutions.

The first problem is to give an interpretation for relatively bounded motion, i.e. a group. There are two possibilities, and we accept both of them. The first is that these groups correspond to galaxies. Of course, since there are no upper or lower limits (other than 1 or $n$ ) for the number of particles belonging to a group, some of the groups simply may be escaping particles. We shall treat them as 'one-particle galaxies'.

It is possible that some of the observed clusters of galaxies are bounded. In this case some of the groups would correspond to 'bounded clusters of galaxies'. Therefore, a circle in Figure 1 could be interpreted as being either galaxies or a bounded cluster of galaxies.

The next motion is where the distance between objects expands like $t^{2 / 3}$. That is, particles not belonging to the same group will separate like $t^{2 / 3}$ (or faster). It is easy to show that this separation can be translated to the centers of mass of the groups; namely, the centers of mass of the groups separate like $t^{2 / 3}$. For purposes of identifica- 


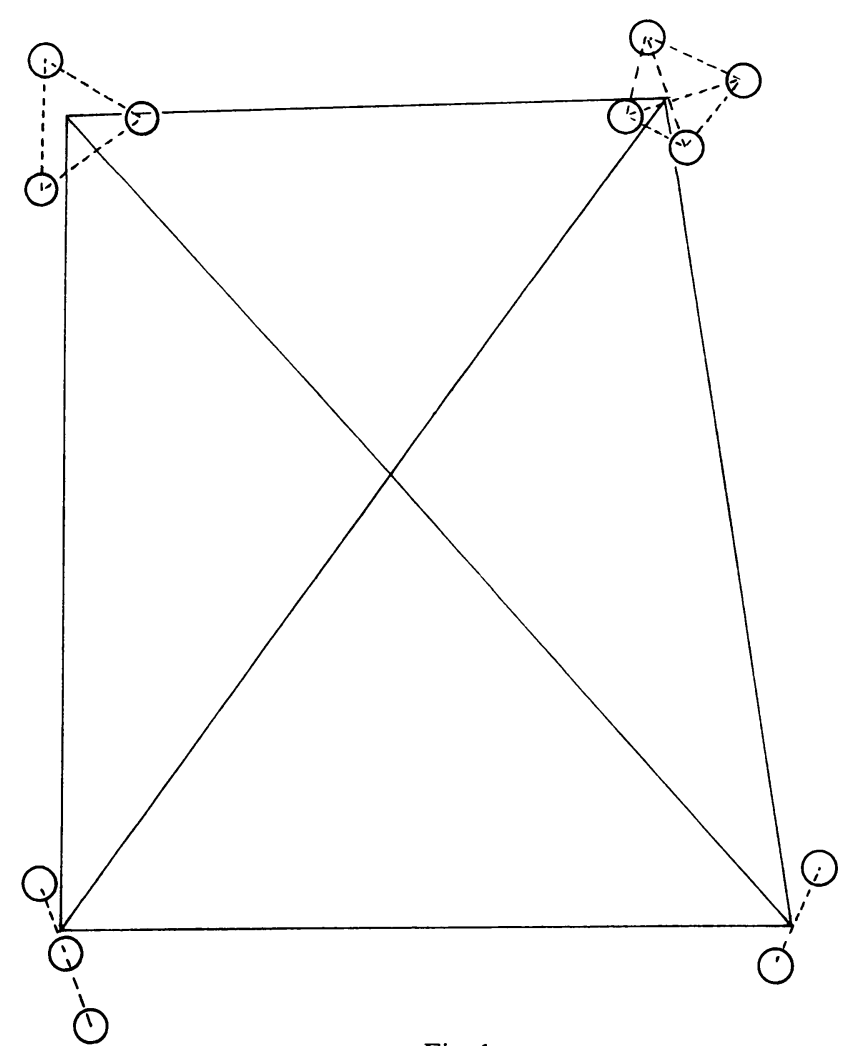

Fig. 1.

tion we denote motion in this setting as subsystems. It was shown in Paper 1 that in subsystems the velocities between the centers of mass of the groups go to zero like $t^{-1 / 3}$.

If the groups are interpreted as being galaxies, then a second type of cluster of galaxies appears. In this case they are galaxies where the distances between them are separating like $t^{2 / 3}$. That is, in this case the system is disintegrating, and the cluster of galaxies is a subsystem of individual galaxies. Since the magnitudes of the velocities become very small, it would seem to be observationally difficult, if not impossible, to distinguish this case from a 'bounded' cluster of galaxies.

A more interesting and intriguing case is when one or more of the groups in a subsystem is a bounded cluster of galaxies. Indeed, we would obtain a cluster of clusters of galaxies, or an hierarchical cluster of galaxies. Again, since the velocities between the centers of mass of the groups are very small, it would seem to be observationally difficult to distinguish this case from a bounded cluster of galaxies.

Of course, there remains the final possibility that a subsystem has only one bounded object. Under the first interpretation of bounded motion, this would yeild a field galaxy. Under the second interpretation, this would give an isolated cluster of galaxies.

The remaining type of motion between particles has the distance between objects 
expanding like $t$. In the same fashion as above, this can be described in terms of the centers of mass of the subsystems separating like $c t$ where $\mathbf{c}$ is a constant vector whose value depends on the choice of the subsystems. Thus, this gives us a description of the expansion between clusters of galaxies. Since $t^{2 / 3} / t \rightarrow 0$ as $t \rightarrow \infty$, even a disintegrating cluster of galaxies given by the above subsystem interpretation will, for all time and from large distances, remain as an observational entity. As will be seen in the next section, even the configuration will remain the same!

This completes the picture for large values of $t$. However, for an evolving system there is a second possible interpretation for this expansion which is asymptotic to $t$. Quite simply, the system may not be old enough to show the sharp distinctions between the different possible types of motion given in Figure 1. That is, since $t$ is 'small', $t^{2 / 3} / t$ is still 'large' for this particular cluster. Consequently, there remains the possibility that a cluster of galaxies is a new system where the rate of expansion of some of the galaxies is like $t$, that is, the bounded motion is separating like $t$, but the act of disintegration occurred recently. Note that with this interpretation, over long periods of time the cluster of galaxies would not remain as an 'observational entity', but it would evolve either into new clusters or field galaxies as described above. The time span necessary for this to occur is, of course, mach too large to be of practical 'observational' value and hence the use of quotation marks. But it is important in the study of the evolution of the system and the 'stability' of clusters of galaxies to distinguish between these two types of disintegrating clusters.

\section{Central Configurations and Subsystems}

A surprising fact about subsystems is that the centers of mass of the groups must tend toward the vertices of well defined (expanding) configurations which depend on the masses of the groups. Thus, clusters of galaxies forming a disintegrating cluster of galaxies, or an hierarchical cluster of galaxies, would tend to form configurations which depend on the masses of the galaxies.

Assume that we have a subsystem which has $p$ groups. In other words, assume that we have $p$ galaxies, $p$ bounded clusters of galaxies or $p$ combinations of these two classes. Let $M_{s}$ be the total mass of the sth group, $s=1,2, \ldots, p$, and let $\mathbf{r}_{s}$ be the vector position of the center of mass of the sth group relative to the center of mass of the subsystem.

It turns out that the expansion $t^{2 / 3}$ is slow enough to allow the attracting force due to the other groups to strongly influence the eventual direction of $\mathbf{r}_{\boldsymbol{s}}$. This forces the position vector and the gravitational force vector to tend to line up along the same line. In fact, as shown in Paper 2, they tend to do so in such a fashion that if $G$ is the gravitational constant, then

$$
\lambda M_{s} \mathbf{r}_{s}+\sum G M_{s} M_{k}\left(\mathbf{r}_{k}-\mathbf{r}_{s}\right) / r_{k s}^{2}=\mathbf{e}_{s}, \quad s=1,2, \ldots, p,
$$

where $\lambda$ is proportionality constant independent of subscript $s$, and $\mathbf{e}_{s}$ is an error term which goes to zero faster than $\lambda\left|\mathbf{r}_{s}-\mathbf{r}_{j}\right|$ as $t \rightarrow \infty$. It follows from Paper 2 that $\lambda=\frac{2}{9} t^{-2}$ and $\mathbf{e}_{s}$ is such that $t^{4 / 3} \mathbf{e}_{s} \rightarrow \mathbf{0}$ as $t \rightarrow \infty$. 
Now it turns out that the centers of mass of the groups tend to the vertices of configurations defined by Equation (1) when the error term is set equal to zero. These configurations, known as central configurations, are determined by the masses of the groups (see Wintner, 1941). That is, this equation shows that the centers of the mass of the groups must form an expanding central configuration. For example, if $p=3$, then the configuration must be either an (expanding) equilateral triangle or one of the Euler straight line solutions where the relative distances are determined by the value of the masses. The main point is that for clusters of galaxies defined by subsystems of individual galaxies, the galaxies must tend to form some central configuration. The same statement holds for an hierarchical cluster of galaxies.

The case $p=3$ is misleading by the regularity of the configurations. For $p>3$ the configurations are by no means so regular and obviously recognizable. In fact, only some of them are known.

The fact that the subsystems tend to define configurations could possibly be exploited in several ways. It could be used (at least theoretically) to distinguish the various types of clusters described in the previous section. The problem is to determine some way to differentiate between a bounded and disintegrating cluster of galaxies. If the masses and the configurations of the groups come 'close' to satisfying Equation (1), then I suggest that there would be additional reason to suspect that the cluster is disintegrating and that the expansion is like $t^{2 / 3}$.

Secondly, if it is known (or suspected) that a cluster of galaxies is defined in terms of a subsystem, and if the masses of the galaxies and the configuration they form (in three-dimensional space) is known, then by substituting these values into Equation (1) it can be determined whether the cluster is complete. If the equation is not satisfied, then the cluster is not complete. A computer search scheme could be devised to determine the necessary location of additional masses needed to satisfy the central configuration equations.

Finally, if the system is known to be complete, and the configuration formed by the groups is known, then in some cases the central configuration equations can be solved for the individual masses of the groups up to certain proportionality parameters. (Unfortunately, this last approach will not work in all cases. For example, it fails in the equilateral triangle case previously cited.)

The observational difficulty with the above program is that the configuration must be known in three dimensional space; whereas, observationally only the projection of the configuration can usually be determined. (Because of these and other problems I hesitate to even attempt to relate the above to such observed objects as the "quadrangles'.) Also, the number of galaxies in a cluster may be so large as to make this theoretical scheme impractical. However, these questions are outside the realm of my experience, and I leave them to other investigators.

\section{Hubble's Constant and Quasars}

As in the case with most models of the Universe, the Hubble constant for the $n$-body 
problem assumes a functional form. The function is particularly simple, and it is exactly what one would expect from the solution of the two-body problem. In the case of subsystems it is, $v / r=\frac{2}{3} t^{-1}+0\left(t^{-1}\right)$ where $t$ is time. The term $0\left(t^{-1}\right)$ denotes error terms of magnitude such that $t 0\left(t^{-1}\right) \rightarrow 0$ as $t \rightarrow \infty$. Here $v$ and $r$ are respectively the speed and distance between the centers of mass of two groups in a sub-system. Hubble's constant for clusters of galaxies, or any other objects where the expansion between them is like $t$, would be $v / r=t^{-1}+0\left(t^{-2}\right)$. The term $0\left(t^{-2}\right)$ denotes error terms such that $t^{2} 0\left(t^{-2}\right)$ is bounded as $t \rightarrow \infty$. This functional form for the Hubble constant is in terms of the instantaneous values of velocity, distance, and time. The constant is obtained by substituting the current 'age of the Universe' into the equation. (The reason for the quotation marks about the phrase 'age of the Universe' is that the inverse square force law model does not naturally admit, or define, a starting value for the birth of the Universe. Unless we restrict attention to a set of initial conditions of measure zero (Saari, 1971c), where as we go backward in time we find a point from which all particles were expelled - A newtonian 'big bang' - there is no natural point on the time scale to assign the origin.)

Can the Hubble relationship be violated ? Expressed in other words, is there anything in the dynamics of the $n$-body problem which would correspond to quasars, in particular to the 'local' hypothesis? That is, is there motion where the velocities are so large that the value of $v / r$ is much larger than accepted values for Hubble's constant? Unfortunately the answer must be in the form - maybe. There is a possible motion in the $n$-body problem, called oscillatory motion, which does satisfy the condition that at different times the velocities of some of the bodies may be too large to satisfy a Hubble's constant relationship. This may or may not be the dynamical equivalent of quasars.

There are several problems involved. The first is the very difficult mathematical problem of existence. Such motion has been shown to exist (Sitnikov, 1960) in only a very specialized setting where the velocities of the particles are nowhere near the required magnitudes needed to allow a dynamical explanation for the red shift of quasars and a local distance of the object. However, the question of existence is so difficult that the Sitnikov example, by its physically timid (but mathematically clever and technically difficult) construction, would be expected to yield same results, namely low velocities. (It also may be a characteristic of the three-body problem, rather than the $n$-body problem.) Theoretically, other types of oscillatory motion may exist where the velocities must be very large. While I have been unable to prove the existence of such motion where the velocities are of the required magnitude, I have likewise been unable to show that it does not occur!

Although the existence question still remains open, several properties of this motion are known, and they will be presented here. This motion is characterized by an oscillating behavior between particles. Namely, three masses $m_{j}, m_{i}, m_{k}$ can be found such that (i) $\lim \sup \left(r_{i k}+r_{k j}\right)=\infty$, (ii) $\lim \sup \left(r_{i k} / r_{k j}\right)>0$, and (iii) $\lim \inf \left(r_{i k} / r_{k j}\right)=0$.

Any particle $m_{s}$ with the property that index $s$ can be substituted for one of the above indices is said to participate in the same oscillatory motion.

We distinguish two cases. The first is where there is some distance between particles 
such that $\lim \sup \left(r_{k j} t^{-1}\right)=\infty$. This motion is the cause of the difficult question of escape. This motion is also related to the question of non-collision singularities. For additional discussion we refer the reader to Saari (1973b).

The second type of oscillatory motion is where $\lim \sup \left(r_{k j} / t\right)=0$ for all particles $m_{k}, m_{j}$ participating in the same oscillatory motion. In this case the particles defining the same oscillatory motion would appear in the sketch of the evolving Newtonian universe in the same way that a subsystem does. The centers of mass of subsystems and oscillatory motion would separate like a constant vector multiple of $t$. Therefore, if oscillatory motion did correspond to quasars, then theoretically we would expect to find quasars separated from the clusters of galaxies as described in Section 2, that is, at least for large values of time.

Additional research has indicated, but not proved conclusively, that the particles with large velocities are associated with larger masses (or clusters of mass). In fact this seems to be the mechanism which allows such large velocities to occur at local distances. The particles commute between the larger masses, which may be quite far apart, picking up velocity with each close passage. Observationally this means that if quasars correspond to oscillatory motion, then some of the quasars should be observed near larger objects.

While the above two paragraphs seem to agree with some of the observed characteristics of quasars (Arp, 1971), there is nothing in this dynamical description which would explain the lack of observed blue shifts for quasars. (Personally, I am skeptical that this is an explanation for quasars. However, it does yield a model employing known physical principles which may [or may not] admit distances much closer than those indicated by their red shift and Hubble's constant.)

A discussion of oscillatory motion in the three- and $n$-body problems can be found in Saari (1973b). It is shown here that in the three-body problem oscillatory motion has its expansion bounded above by a constant multiple of $t^{2 / 3}$.

The remaining class of motion is called pulsating motions. Its definition and some of its properties can be found in Paper 1. It can be shown that the distances between particles separate no faster than $0\left(t^{2 / 3}\right)$. (This is true also for the second type of oscillatory motion.)

Therefore, if pulsating motion exists, it would play the same role as a group or 'galaxy' in the above sketch of the Universe.

\section{The Virial Theorem}

For at least the last decade the virial theorem approach to determine the masses of galaxies has been under attack. For example, it is known that unstable systems will give exaggerated values for the masses. With a partial solution of the $n$-body problem available this statement can be derived in a rigorous fashion, and we do so here.

Of course, there are other serious difficulties involved in using the virial approach, such as the need to use the instantaneous values for $U$ and $T$ rather than their time averages. Here $T$ is the kinetic energy of a system and 


$$
2 U=\sum_{i} \sum_{j} G M_{i} M_{j} / r_{i j} .
$$

(In Paper 2 the assumption that the instantaneous values and the time averages agree over a period of time was shown to imply that $T=-h$ and $U=-2 h$ for all time. Here, $h$ is the total energy of the system. Furthermore, it is now known (Saari, 1973b) that if $T$ and $U$ are equal to constants over a period of time, then the constants must be respectively $-h$ and $-2 h$ for all time. Finally, it is conjectured that this implies that the motion behaves like a rigid body. It is true for the three-body problem.)

In studying the effects the dynamics have on the virial theorem approach to mass determination, we shall ignore all other approximations. That is, we shall assume that all additional approximations leading to the simplification of the problem can be made without error. With this assumption the error in the value of the masses introduced by the hypothesis $2 T=U$ will be examined under the different dynamical interpretations of clusters of galaxies.

Assume first that we have a bounded cluster of galaxies and that the masses in the definition of $T$ and $U$ are the masses of the galaxies. Then by Paper 2 and Section 2 of this note, $T-U$ is eventually negative. Now, the way the masses are determined goes as follows. Depending on the technique employed, certain assumptions are introduced to obtain the ratios of masses of the galaxies. This reduces the problem to a quasi-binary problem. That is, the 'virial' equation is changed from $2 T=U$ to $2 V^{2}=2 M / R$, where $V$ and $R$ are defined as

$$
V^{2}=2 T\left(\sum M_{s}\right), \quad R=\left(\sum M_{s}\right)^{2} / 2 U
$$

$M$ is the total mass of the system, and it is the unknown. Let $M^{*}$ be the value of the total mass obtained from the assumption $2 T=U$, and let $M$ denote the correct value of the total mass. That is $M^{*}=2 R V^{2}$.

The same assumptions used in the above reduction apply to the energy relationship converting $T-U<0$ to $V^{2}-M / R<0$, or $M>R V^{2}$. That is, $M^{*}<2 M$. Consequently, if the system is bounded, then the error introduced by the assumption $2 T=U$ is bounded above by a factor of 2 .

The situation changes if the cluster of galaxies is defined by the subsystem description. It was shown in Paper 1 that in this case the energy relationship assumes the form $T-U \rightarrow 0$. That is, $V^{2}-M / R \rightarrow 0$ or $M^{*}=2 R V^{2} \rightarrow 2 M$. Hence the error introduced by $2 T=U$ in this setting is approaching a factor of 2 .

Finally, if a cluster of galaxies is defined by an expansion like $t$, or a mixture of expansions of the type $t^{2 / 3}$ and $t$, then $T-U$ approaches a positive constant. Hence, $V^{2}-M / R \rightarrow 2 H / M>0$. In this setting, $M^{*}=2 R V^{2} \rightarrow 2 M+2 H R / M$. Since $R$ expands like $t$, it follows that the error in the computed value $M^{*}$ will become at least twice as large as the correct value $M$. Actually the error will become arbitrarily large with the actual value of the error depending on the value of $H$ and the age of the system.

\section{Improbability of Certain Motions}

In dynamical astronomy and celestial mechanics there are several statements to the 
effect that the set of initial conditions leading to a certain type of behavior has measure zero. That is, in a measure-theoretic sense it is mathematically improbable that such motion will occur. Statements of this type include collisions in the $n$-body problem (Saari, 1971c, 1973a), capture and escape (Chazy, 1922; Hopf, 1930), and motion which includes $t^{2 / 3}$ expansions in the two- and three-body problems. The next step is to assert that such motion probably does not occur in the Universe; consequently, it can be ignored.

While this last step is well understood to be incorrect, the argument periodically reappears in the literature. Therefore, we outline here some of the objections to this statement. The ideas advanced here are strongly motivated by a paper by Schwarz (1962) aptly titled 'The Pernicious Influence of Mathematics on Science'.

For the above assertion to be correct there remains the important step and basic problem of showing how the mathematical probability of an event corresponds to the observational (or experimental) probability of the same event. They need not be the same! The major problem is, of course, that while for technical reasons the Lebesgue measure is a natural measure to use in the study of the $n$-body problem, there is no reason to believe that nature is laboring under the same technical constraints. She may be fooling us with a different distribution function.

Even if the Lebesgue measure is the proper measure, the Universe may exhibit prejudices for certain initial conditions which are imposed upon it by different physical constraints due to conditions of its creation, etc. To illustrate this further, recall that in the $n$-body problem the set of initial conditions corresponding to zero angular momentum has measure zero. However, if the Universe does date its birth from some sort of 'big bang', then as the position vectors are traced backwards in time $(t \rightarrow 0)$ to their common origin we have a mathematical collapse of the system, and all the position vectors tend to zero. This means that the total angular momentum is zero. Thus the motion is contained in a set of measure zero. (This could be viewed as a 'collision' at some past time. Again this means that we are restricted to a set of measure zero.) Now, phenomena peculiar to zero angular momentum would be mathematically improbable but they may be observationally and physically abundant. (Indeed, we should be using conditional probability.)

In other words, the constraints given by the creation of the Universe, if simply its survival to the present date, may actually restrict the initial conditions of the mathematical model to sets of measure zero or lower dimensional manifolds. Consequently, certain motions cannot be ruled out of the real world simply by mathematical probabilistic statements, they can only be ruled out by observational evidence proving that such predicted behavior does not, has not, and will not occur. Hence in dynamical astronomy the problems of capture, collision, etc. are solved in a mathematical probabilistic sense, but they must be considered open questions in a physical probabilistic sense! (The collision problem uses point masses, hence it would not apply for this reason alone.) 


\section{Comments}

The above description given in Sections 2-5 depends on the behavior of solutions of the $n$-body problem for large values of time. Clearly our Universe has not reached that advanced age where all particles (stars or galaxies) have committed themselves or displayed their future course of expansion. Some clusters of galaxies may have only recently begun the process of disintegration, and they would not show the clear distinction between the different rates of expansion as indicated in Figure 1. Some groups of particles may at some future time evolve into several galaxies, and each of these might separate from each other. All of this leads to a far more complicated picture than the one indicated in Sections 2 and 3.

Of course, certain characteristics of the expansion would begin to manifest themselves at an earlier stage of the evolution, however, not with the same distinction as will eventually be the case. For example, the configurations discussed in Section 3 depend on the rate of expansion and the masses of the groups. If certain groups are separating from each other like $t^{2 / 3}$, then we would expect these groups to start to form central configurations. (The error of deviation from the correct configuration would be much larger than will be the case in the future.) This is independent of the fact that at some time in the future some gathering of particles may separate into two or more smaller groups with separations like $t^{2 / 3}$ or $t$. This is because the force law which gives these configurations, depends on the present location of accumulations of mass - not some future location or future behavior of the masses nor current velocities within the groups.

So although the Universe may be quite young, certain characteristics of the above description may already be displayed, and the above discussion may be of value in interpreting present observations.

Of course, the above is predicated on the unproved assumption that the inverse square force law is a valid approximation to the gravitational forces which governs the motion of the Universe. As was stated in the introduction, the force law used in this note can be quite general, and it is permitted to run the spectrum between the inverse force law and the inverse cube force law with fairly large perturbations permitted. All of this causes only minor modifications in the above discussion. The question still remains, can the gravitational force be approximated by these force laws? While we cannot answer this question, we can at least offer the following corollary. If it can be shown that the dynamics of the Universe cannot now or ever in the future be described in terms of the above qualitative discussion, then it follows that any inverse force law between the inverse and the inverse cube force laws is not dominant for large distances.

Finally, using the fact that the model allows for fairly large perturbations of the gravitational force, a prèjudice among some investigators of dynamical astronomy against parabolic orbits, or in our setting, against motion separating like $t^{2 / 3}$, will be examined. The argument for their position usually centers around two points. The first is that the motion may be improbable in a measure-theoretic sense. The second is that 'small' perturbations disrupt this motion into expansions such as $t$, or into 
bounded motion. Hence, due to their sensitive nature, they would not, in general, survive. That is, one would not expect such motion to exist in the real universe.

The first statement was examined in a more general setting in the previous section. If the second holds, it can have some interesting consequences. As was previously noted, the actual central force law employed can deviate from the theoretical inverse square law by 'almost' as much as the law itself. Viewing these deviations as perturbations of the system, we have that perturbations which do not allow for this expansion are in reality quite significant forces, as significant as the inverse square term itself. If these forces do occur in nature, then they must be studied and understood for they significantly alter our understanding of gravitational forces! (In the same sense, if approximation schemes such as numerical integration cannot obtain these orbits, then the errors introduced must be considered as being quite large in a qualitative sense.)

It may be that oscillatory motion, $t^{2 / 3}$ expansion, or other motions discussed in this note do not occur in the universe; however, this is outside the realm of theory, and it must be observationally determined.

\section{Acknowledgement}

This research was partially supported by NSF contract GP-32116.

\section{References}

Ambartsumian, V. A. : 1961, Astron. J. 66, 536-41.

Arp, H.: 1971, Science 174, 1189-2000.

Chazy, J.: 1922, Ann. Sci. Ecole Norm. 39, 29-130.

Hopf, E.: 1930, Math. Ann. 103, 710-19.

Saari, D. G.: 1971a, Trans. Am. Math. Soc. 156, 219-40.

Saari, D. G. : 1971b, Astrophys. J. 165, 399-407.

Saari, D. G.: 1971c, Trans. Am. Math. Soc. 162, 267-71.

Saari, D. G.: 1973a, Trans. Am. Math. Soc. 181, 351-368.

Saari, D. G. : 1973b, J. Diff. Eq. 14, 275-292.

Saari, D. G. : 1974, SIAM J. Appl. Math. 5.

Schwarz, J.: 1962, in E. Nagel, P. Suppes, and A. Tarski (eds.), Logic, Methodology and Philosophy of Science,

Stanford University Press, Stanford, Calif., pp. 356-60.

Sitnikow, K.: 1960, Dokl. Akad. Nauk SSR 133, 303-06.

Wintner, A.: 1941, The Analytical Foundations of Celestial Mechanics, Princeton University Press,

Princeton, N.J., U.S.A., pp. 274-75.

\section{DISCUSSION}

J. Moser: Can one give a detailed analytic description of the escape, in particular for $n=3$, of a bounded pair is present?

D. Saari: Yes. In this case the escaping particle would correspond to a 'one-particle' galaxy. For $n=3$ it would be possible to obtain a very detailed description.

J. Moser: Do you have a guess which of the asymptotic behaviors described by you is predominant?

D. Saari: I would guess that pulsating motion does not exist. If oscillatory motion with the property $\lim \sup r_{k j} / t=\infty$ does exist, then I would guess that it would be rare, i.e. of measure zero. This last problem is related to the problem of noncollision singularities. 